\title{
Pitfalls of Incomplete Myelography with Thoracic Spinal Lesions
}

\author{
Hart C.M. Cohen and William S. Tucker
}

\begin{abstract}
This paper describes four patients with thoracic spinal lesions in whom the initial clinical presentation was highlighted by complaints in the lower back and lower extremities, in the absence of thoracic spinal or radicular symptoms. Initial myelography, confined to the lumbar region, failed to reveal a cause for the patients' symptoms. Subsequently, diagnostic consideration of a thoracic spinal lesion prompted repeat myelography of the thoracic region which demonstrated a relevant lesion in each case. It is important to visualize the thoracic cord when myelography is performed for the investigation of pain or neurological symptoms in the lower back or lower extremities.
\end{abstract}

RÉSUMÉ: Ecueils associes a une myelographie incomplete en presence de lesions spinales thoraciques Nous décrivons quatre patients avec des lésions spinales thoraciques chez qui la présentation clinique initiale était marquée de plaintes impliquant le bas du dos et les membres inférieurs sans symptômes thoraco-spinaux ou radiculaires. La myélographie initiale limitée à la région lombaire fut négative. Lorsque, plus tard, de nouveaux indices cliniques impliquèrent la région spinale thoracique, une nouvelle myélographie démontra, dans chaque cas, une lésion compatible. Il est donc important de toujours visualiser la région thoracique en myélographie, même si les symptômes sont confinés au bas-dos et aux membres inférieurs.

Can. J. Neurol. Sci. 1985: 12:121-124

Lesions involving the thoracic cord are relatively rare and pain in the lower back or lower extremities is seldom attributable to a lesion in the thoracic spine (Van Damme et al., 1979). Clinicians may, therefore, fail to consider the possibility of a thoracic spinal lesion and the diagnosis may be missed because of an incomplete myelogram.

We will describe four patients, each with a different pathological entity in the thoracic spine, who presented with pain or neurological symptoms in the lower back and lower extremities. In each instance, myelography was confined initially to the lower lumbar region and failed to demonstrate the relevant abnormality. We wish to emphasize the importance of visualizing the thoracic spine when myelography is performed for investigation of lumbosacral or lower limb symptomatology. This issue, to our knowledge, has not been specifically addressed in the literature; it is analogous, however, to the diagnostic difficulty posed by foramen magnum tumors. It is recognized (Editorial: Lancet, 1973) that the latter occasionally escape detection due to inadequate upward passage of contrast material for myelographic visualization.

\section{CaSe RePorts}

\section{Patient 1}

This 58 year old man presented in March 1980 with a four year history of low back pain radiating down the right lower extremity to the foot. He had been impotent for two years, and had required a cane to walk for one year. He complained of numbness of the right leg.

In 1978 a myelogram had been performed elsewhere, and had suggested a disc herniation at L5-SI on the right side. The contrast material had not been run cephalad to the lumbar spine. The L4-5 and L5-SI levels were explored, but discotomy was not carried out because of heavy epidural bleeding. Later, repeat lumbar myelography, epidural venography, and aortography were performed, but no conclusive abnormality was demonstrated. A right sympathectomy was performed in October 1979 , without benefit.

In March 1980 the patient demonstrated right lower limb weakness. spasticity, and hyperreflexia, and both plantar responses were extensor. There was bilateral sensory loss to all modalities below the knees. A complete myelogram was recommended, and in the supine position, this showed multiple serpiginous defects, less apparent in the prone position, extending from the T8 to T12 vertebral levels, and consistent with a cord arteriovenous malformation (AVM) (figure 1).

In February 1980 the AVM was removed completely through a laminectomy exposure using microsurgical technique. The patient's neurological deficit and pain have persisted, but have not progressed since surgery. 


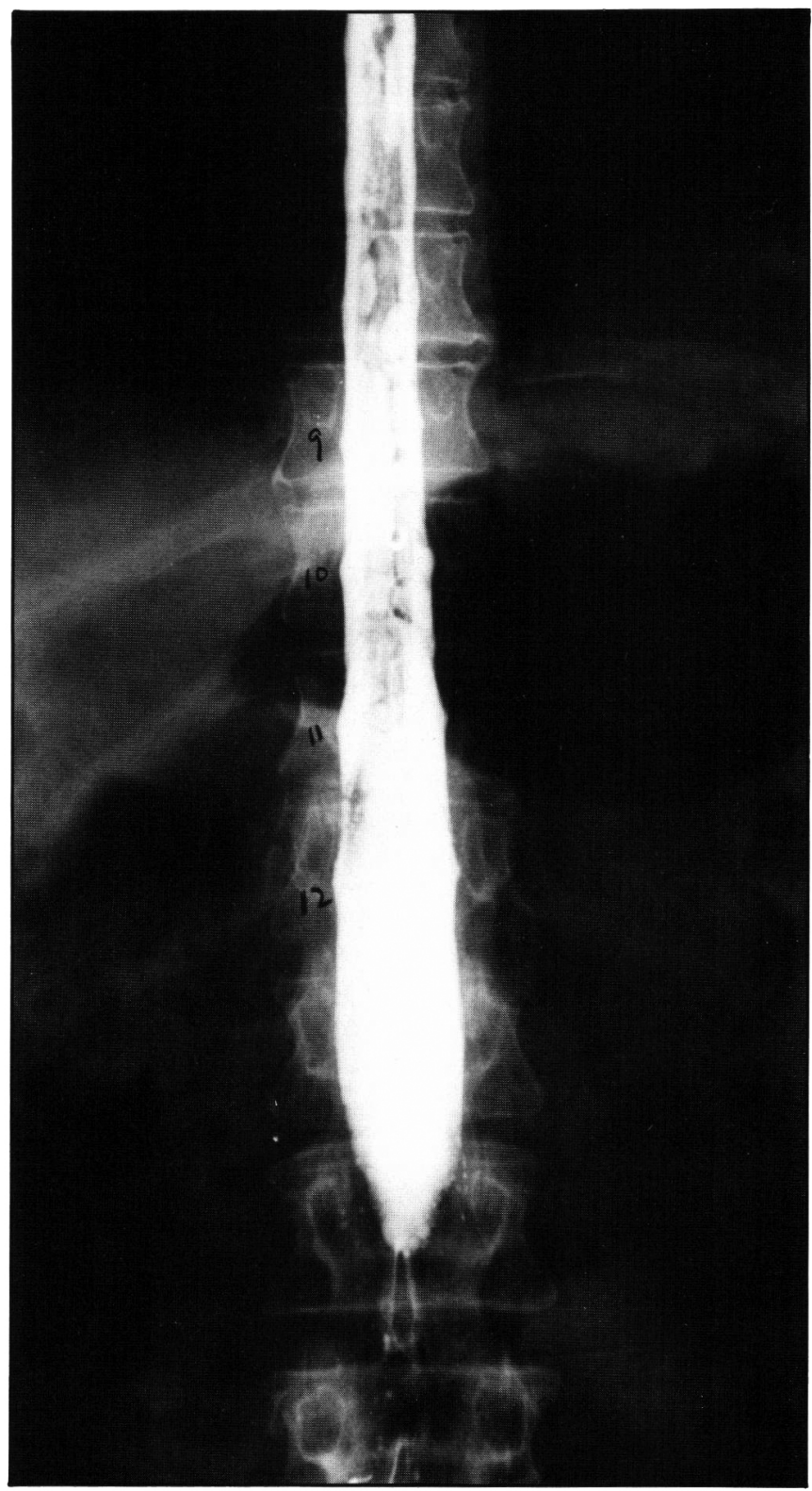

Figure I - Repeat myelogram in Patient I. Multiple serpiginousfilling defects are demonstrated in the thoracic region, consistent with the presence of an AVM.

\section{Patient 2}

In the fall of 1980 , this 67 year old woman developed numbness of the left leg and later the right leg. By the summer of 1981, she complained of burning paraesthesiae of the feet, weakness and stiffness of her legs, and difficulty walking. Bladder emptying became difficult, and there was decreased awareness of bowel evacuation. Plain spinal X-rays showed thoracolumbar rotoscoliosis and degenerative lumbar disc disease. A myelogram limited to the lumbar segments was carried out at a community hospital in January 1982, and revealed only a small disc protrusion at L4-5 on the left. The patient experienced increasing back pain and neurological deficit, and was referred to our institution in May 1982.

Examination revealed a spastic paraparesis with hyperreflexia and bilateral Babinski responses. There was profound posterior column sensory loss in both lower limbs, and milder spinothalamic deficit, worse on the left side, below the knees. A complete myelogram was recommended, and demonstrated a total block at $\mathrm{T} 7$, compatible with an intradural extramedullary lesion (figure 2).

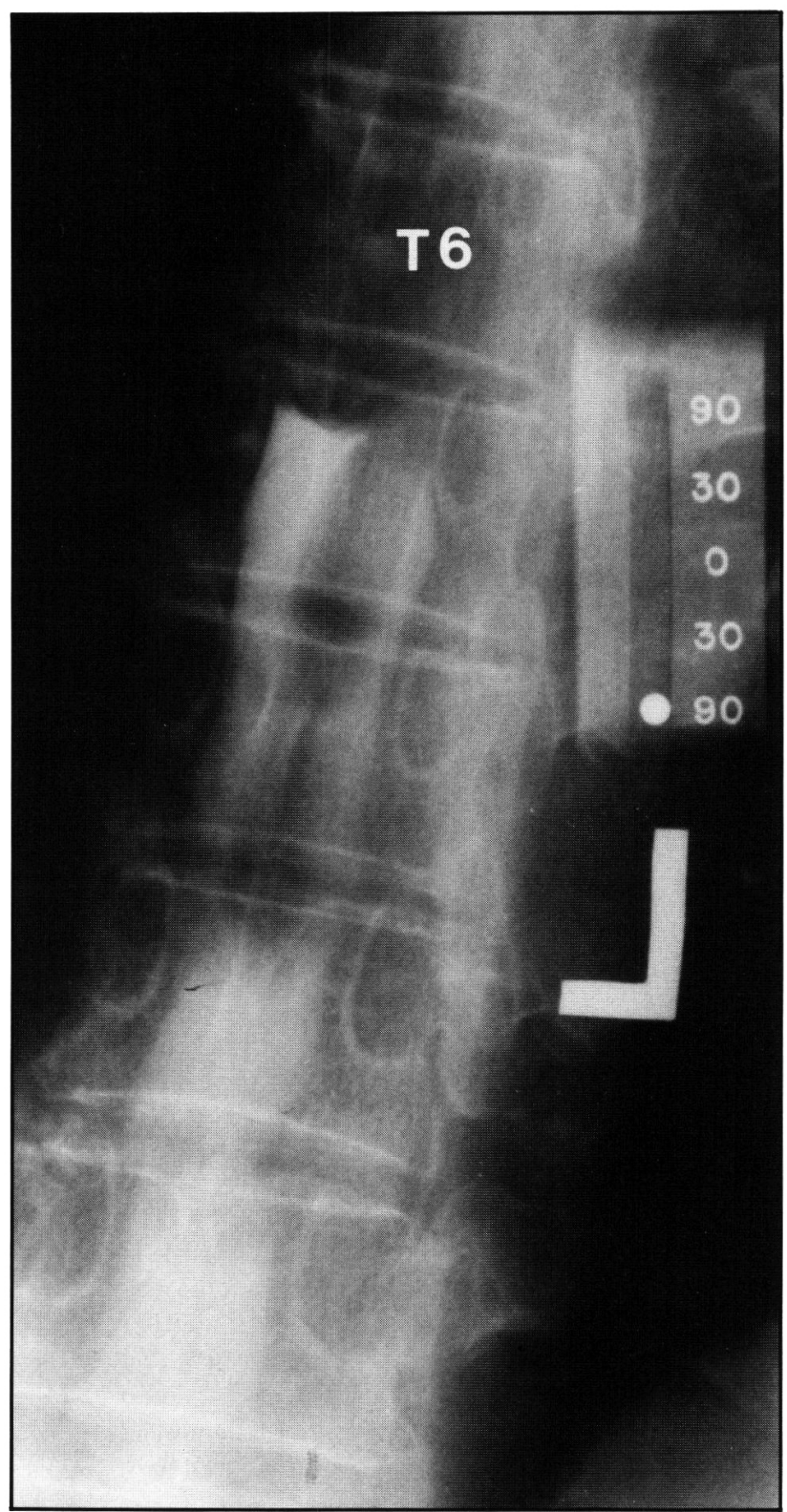

Figure 2 - Repeat myelogram in Patient 2. This demonstrates a complete block at the level of the T6-T7 intervertebral disc, compatible with an intradural extramedullary lesion.

Subsequently a psammomatous meningioma was resected through a thoracic laminectomy, using the operating microscope. At recent follow-up, the patient was free of pain, and described normal bowel and bladder control. She walked well, with very minimal spasticity, and there was only minor sensory loss in the feet.

\section{Patient 3}

This 64 year old man was seen at another center in May 1983, with a three year history of left leg pain, and a three month history of lower back and buttock pain. Just prior to his admission to a community hospital, increasing leg weakness, predominantly on the left, had forced reliance on one and then two canes. At that time the physical signs included wasting of the left quadriceps, with left hip, knee, and ankle weakness. The left knee reflex was absent. There was reduced sensation over the left thigh, lateral calf, and dorsum of the foot. A myelogram was restricted to examination of the lumbar segments (figure $3 \mathrm{~A}$ ). The $\mathrm{CSF}$ protein was markedly elevated, but no radiologic lesion was demonstrated. 

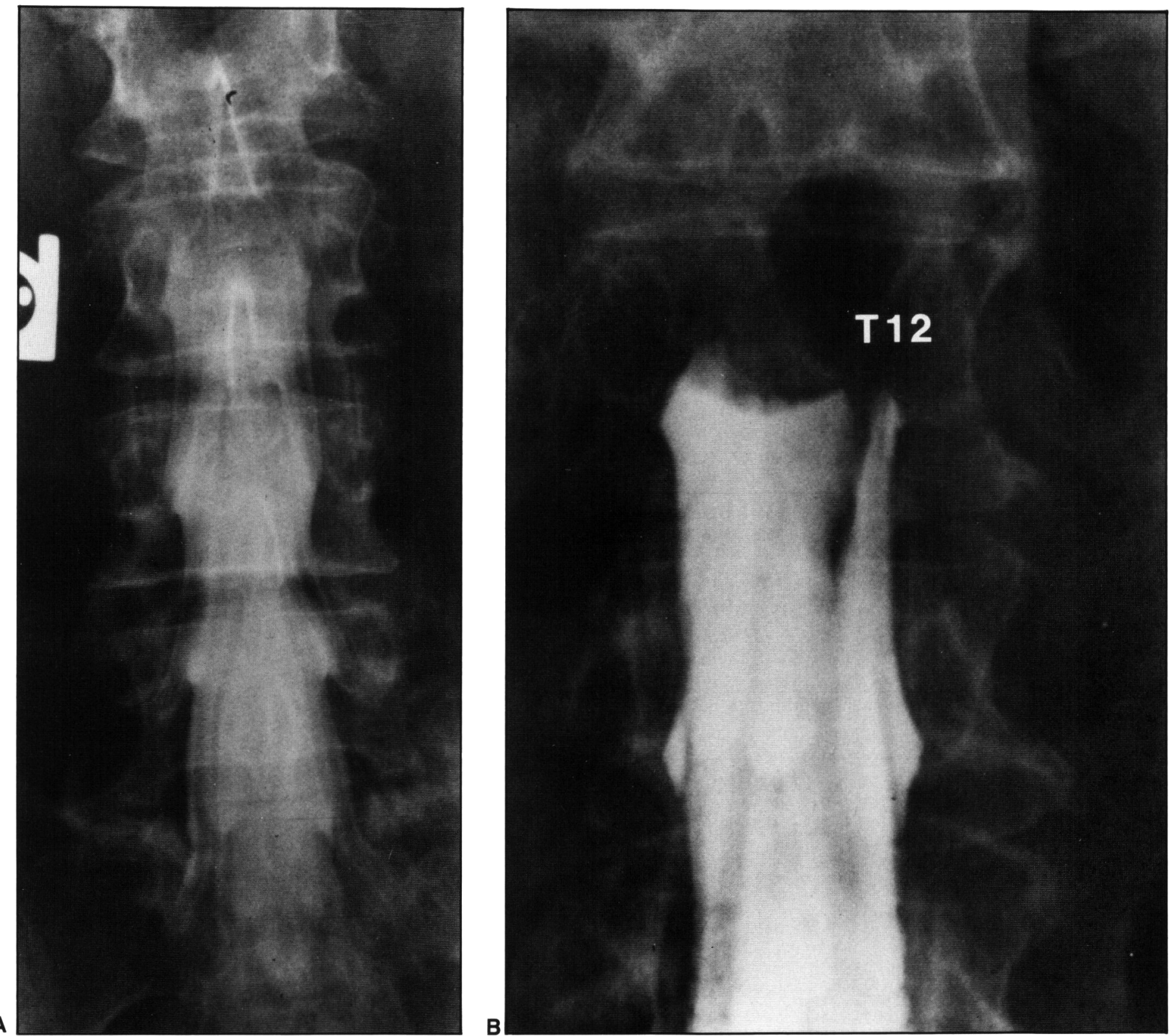

Figure 3(A) - Initial myelogram in Patient 3, in which contrast material was confined to the lumbar region. No abnormality is demonstrated.

(B) Repeat myelogram. A complete block at vertebral level T12, compatible with an intradural extramedullary lesion, is demonstrated.

Over the subsequent two weeks, the patient deteriorated markedly, and we were asked to assess him. The examination revealed profound paraparesis, worse on the left, where there was no movement at or distal to the knee. The lower limbs were hyperreflexic, with bilateral extensor plantar responses. There was dense posterior column sensory loss in the lower extremities, and an indefinite sensory level to pinprick at T12 bilaterally. A complete myelogram was recommended, and showed a complete block at T12 due to an intradural extramedullary lesion (figure 3B).

In June 1983 a laminectomy of $\mathrm{T} 11$ and $\mathrm{T} 12$ was performed, and using the operating microscope, a yellowish cystic schwannoma was resected from the dorsum and left side of the cord. The lesion arose from a dorsal sensory rootlet, which was sacrificed. The patient made a good recovery, and was able to resume work and cross-country skiing by December 1983.

\section{Patient 4}

This 53 year old man developed pain in the left groin in October 1976, and complained of weakness of the left lower extremity during walking.
Exploration for a left inguinal hernia and subsequent division of the left lateral femoral cutaneous nerve failed to provide relief. A myelogram was performed elsewhere, but only the lumbar segments were examined. Lumbar spinal stenosis was demonstrated, and the patient was referred to our institution for surgical consideration.

Examination revealed increased tone in the lower extremities, and bilateral extensor plantar responses. A repeat myelogram to visualize the remainder of the cord was strongly recommended, but the patient refused, and was subsequently discharged. Eight months later, in March 1978 , the patient returned, barely able to walk with a cane, and had marked spasticity of the lower limbs. There was a sensory level to pinprick at T12 on the left, and the patient complained of severe pain radiating to the left calf. A repeat myelogram demonstrated a major defect at the T11-12 disc level, and a diagnosis of thoracic disc prolapse at that level was made.

A right costotransversectomy was carried out, and the T11-12 disc removed. The strength of the left lower limb improved, allowing resumption of work for several years. However, persisting spasticity 
and painful numbness of the left thigh, with analgesic dependence, led the patient to accept an early retirement from his work.

\section{Discussion}

All four patients in this series presented with lower extremity pain and/or paresthesiae, lower extremity weakness and hyperreflexia, sensory impairment, and gait disturbance. Three patients had visceral symptoms and two complained of low back pain. In each instance, initial myelography was confined to the lumbar region. Clinical suspicion of an abnormality in the thoracic spine prompted repeat myelography which demonstrated a lesion in the thoracic region in each case. Operative findings and pathological examination revealed the following lesions in the four patients: arteriovenous malformation, meningioma, schwannoma, and herniated intervertebral disc.

On clinical grounds, it is frequently difficult to distinguish between thoracic disc herniation and neoplasm (McAllister and Sage, 1976; Love and Schorn, 1965), or to differentiate among the various spinal cord tumours (Obayashi et al., 1980). This difficulty has less clinical significance than the recognized possibility of neglecting altogether a diagnosis of thoracic spinal lesion, whatever the etiology (Bull, 1953; Love and Schorn, 1965). Because thoracic disc protrusions tend to be central, they, in addition to spinal cord tumours, may present with vague, non-radicular symptomatology (Patterson and Arbit, 1978; McAllister and Sage, 1976; Albrand and Corkill, 1979; Love and Schorn, 1965; Arseni and Nash, 1960). This, together with absent or indefinite neurological findings, may result, initially, in a misleading clinical picture. In our patients, the absence of radicular thoracic pain, and the prominence of complaints in the lower back and lower extremities prompted suspicion of a lumbar lesion. Indeed, Signorini et al. (1979), in a report of nine cases of herniated thoracic intervertebral discs, included a patient with central disc protrusion at T9-T10 whose initial symptom was left-sided sciatica present for one year.
Love and Schorn (1965) included lumbar disc protrusion among the misdiagnoses entertained in a series of patients with herniated thoracic discs. Awareness of this diagnostic problem should alert the clinician to keep in mind a diagnosis of thoracic spinal lesion. When performing a myelogram for the investigation of low back pain and lower extremity problems, adequate visualization of the thoracic spine must always be undertaken.

With the availability of epidural venography and C.T. scanning for the investigation of spinal lesions, clinicians will have to be persistently vigilant to avoid misdiagnosis of the type of uncommon but surgically remediable lesion described here. This is especially true for intradural lesions, and those cephalad to an expected lumbar spinal site.

\section{REFERENCES}

Albrand OW, Corkill G (1979) Thoracic disc herniation: treatment and prognosis. Spine 4: 41-46.

Arseni C, Nash F (1960) Thoracic intervertebral disc protrusion: a clinical study. J. Neurosurg. 17: 418-430.

Bull JWD (1953) Spinal meningiomas and neurofibromas. Acta Radiol. (Stockh.) 40: 283-300.

Editorial (1973) Missed foramen-magnum tumours. Lancet ii: 1482.

Love JG, Schorn VG (1965) Thoracic-disk protrusions. JAMA 191: 627-631.

McAllister VL, Sage MR (1976) The radiology of thoracic disc protrusion. Clin. Radiol. 27: 291-299.

Obayashi T, Furuse M, Nakama M (1980) Radionuclide angiography of vascular lesions of the spinal cord: its efficacy in selecting patients for spinal angiography. Arch. Neurol. 37: 572-574.

Patterson RH Jr, Arbit E (1978) A surgical approach through the pedicle to protruded thoracic discs. J. Neurosurg. 48: 768-772.

Signorini G, Baldini M, Vivenza C, Princi L, Tonnarelli GP (1979) Surgical treatment of thoracic disc protrusion. Acta Neurochir. (Wien) 49: 245-254

Van Damme W, Hessels G, Verhelst M, Van Laer L, Van Es I (1979) Relative efficacy of clinical examination, electromyography, plain film radiography, myelography and lumbar phlebography in the diagnosis of low back pain and sciatica. Neuroradiology 18: 109-118. 